\title{
An Alternative Model of Proton and Neutron
}

\author{
Arend Niehaus \\ Retired, Utrecht University, Utrecht, The Netherlands \\ Email:aniehaus@outlook.de
}

How to cite this paper: Niehaus, A. (2020) An Alternative Model of Proton and Neutron. Journal of Modern Physics, 11, 285-293.

https://doi.org/10.4236/jmp.2020.112017

Received: January 21, 2020

Accepted: February 17, 2020

Published: February 20, 2020

Copyright (c) 2020 by author(s) and Scientific Research Publishing Inc. This work is licensed under the Creative Commons Attribution International License (CC BY 4.0).

http://creativecommons.org/licenses/by/4.0/

\begin{abstract}
Based on a model of fermions which implies a model of photons, a model of the neutron is constructed by merging two photons of equal energy propagating in opposite directions. The fermion model is outlined, and the merging of two photons is described in detail. The radius of the neutron obtained in this way is $R_{n}=0.84008 \ldots \mathrm{fm}$. This value is four times the reduced Compton wavelength of the neutron. Assuming the same model for the proton, one obtains a value of $R_{p}=0.84123 \ldots \mathrm{fm}$, which agrees with the most recent experimental value for the charge radius of the proton within the given limits of error. The neutral charge of the neutron is reproduced, and the positive charge of the proton follows within the model, if the proton is formed via the anti-neutron by losing one electron. $S= \pm \hbar / 2$, and zero dipole moment, is also reproduced for proton and neutron. Further, a value of the magnetic moment of the neutron of $\mu=-2.00 \mu_{N}$ ( $\mu_{N}$ : nuclear magnetic moment), and of the proton of $\mu=2.666 \ldots \mu_{N}$ is predicted. The deviation by ca. $5 \%$ from the recommended respective values of $\left(-1.9130 \mu_{n}\right)$, and $\left(2.793 \mu_{n}\right)$ is ascribed to the (g-2)-anomaly. Finally, the relation of the model with the established description of the nucleons in terms of three quarks bound by gluons is shortly discussed.
\end{abstract}

\section{Keywords}

Quantum Physics, Modeling of Nucleons, Classical Probability

\section{Introduction}

The fermion model developed in three recent publications [1] [2] [3], is used in the present paper to construct a model of the nucleons. In paragraph 2, it is outlined. Qualitatively, it describes particles in terms of paths of a quantum around a fixed point in space. The position of the particle is the position of this point, and its mass is the localized energy of the motion. It takes an observation time of at least one period of the periodic motion of the quantum until the particle "ex- 
ists" as an observable entity. This time is very short, and depends as $1 / \mathrm{m}$ on the mass of the considered fermion. In case of the electron it is on the order of the period of the well-known "Zitterbewegung" [4] [5] [6]. For longer observation times the particle described in this way has internal structure, and therefore can own properties. Properties of the particle at rest can be obtained from the stationary spatial density distribution of the quantum, which reflects the topology of its path. Mass, size, spin, and magnetic moment of the electron have been correctly described in this way. For a description of properties of the moving particle, a more detailed use of the motion of the quantum in space and time is necessary. The emergence of the property "de Broglie wavelength" and the implied interference phenomena of the moving particle have also been demonstrated to be a natural consequence within the model [3].

Similar models as the one developed in [1] [2] [3] have been proposed and discussed earlier in the literature [see f.i., [7] [8] [9] [10]]. What we intend to prove in this paper, is the fact that, our fermion model, sketched above qualitatively, allows to construct nucleons and to calculate their properties. In paragraph 2, we describe the fermion model in sufficient detail to demonstrate its application to the construction of the nucleons. In paragraph 3, the "merging" into nucleons is outlined, and results are reported. In paragraph 4, we summarize the results and address shortly the question of a relation between the proposed model and the established theory.

\section{The Fermion Model}

The basic quantity determining the spatial distribution is the reduced Compton wavelength

$$
L=\hbar / m c,
$$

with $(\hbar)$ being the quantum of action, $(m)$ the mass of the fermion, and $(c)$ the velocity of light. The distribution of the quantum in a topology that characterizes a fermion, arises when a ring of radius $(L / 2)$ containing the quantum is rotated around an axis that lies in the plane of the ring, and goes through one point on the ring (see Figure 1).

The population probability of the quantum on the ring is uniform. The possible positions of the quantum form the surface of a torus (see Figure 2). The positions are described by the position vector

$$
\boldsymbol{r}=L\left\{\cos ^{2}\left(\frac{\varphi_{t}}{2}\right) \cos \left(\varphi_{c}\right), \cos ^{2}\left(\frac{\varphi_{t}}{2}\right) \sin \left(\varphi_{c}\right), \sin \left(\varphi_{t}\right) / 2\right\}
$$

The angles $\left(\varphi_{c}\right)$ and $\left(\varphi_{t}\right)$ are, respectively, the azimuth-, and the torus angle. The probability density on the surface of the torus is independent of $\left(\varphi_{c}\right)$, and its dependency on the torus angle $\left(\varphi_{t}\right)$ is determined by the condition that the probability on the ring is uniform (Figure 1). The probability for the position vector to point into a certain area $\left(\mathrm{d} \varphi_{c} \mathrm{~d} \varphi_{t}\right)$ thus becomes simply $\rho=1 /\left(4 \pi^{2}\right)$, independent of the angles. Averages of functions of the position vector, $F(\boldsymbol{r})$, can 


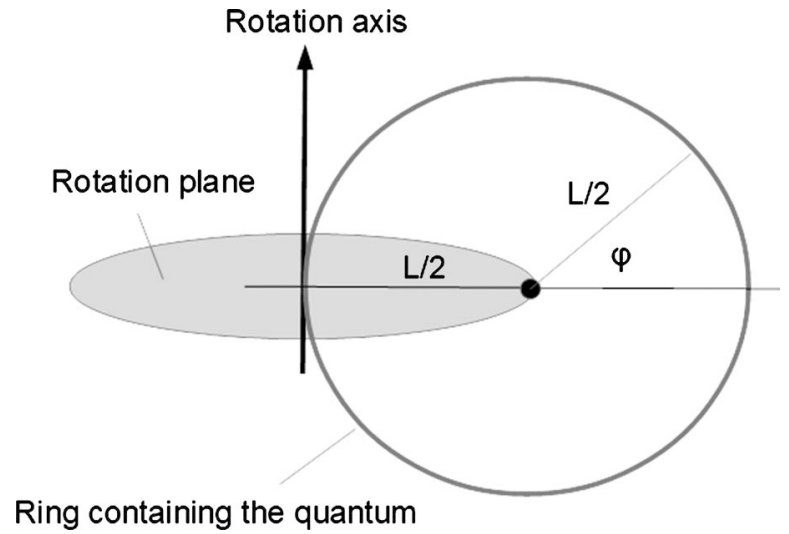

Figure 1. The figure shows the plane containing the quantum. The quantum is distributed with constant probability density on the ring. The plane containing the ring rotates around the rotation axis in the way shown in the figure. The rotation frequency is $\omega_{c}=$ $c /(L / 2)$, and the center of the ring has speed (c). The angle $(\varphi)$ in the figure is the torus angle $\left(\varphi_{t}\right)$ used in the text, and the angle defining the angular position of the ring is the angle $\left(\varphi_{c}=\omega_{c} t\right)$ used in the text.

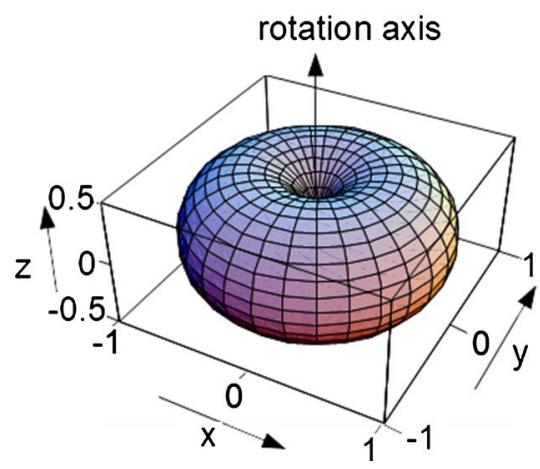

Figure 2. The possible positions of the quantum during its cyclic motion around the rotation axis. The coordinates are given in units of L. The shape of the entity formed after one period of the "Zitterbewegung" is a torus on whose surface the quantum circles. The radius of the entity, which we identify as the fermion, has a radius $R=L=\hbar / \mathrm{mc}$.

therefore be obtained as the integral

$$
F(r)_{a v}=\left(\frac{1}{4 \pi^{2}}\right) \iint F(\boldsymbol{r}) \mathrm{d} \varphi_{c} \mathrm{~d} \varphi_{t}
$$

The momentum ( $p=m c=\hbar / L)$ perpendicular to the ring (see Figure 1$)$ is ascribed to the quantum, so that its momentum vector becomes

$$
\boldsymbol{p}=\left(\frac{\hbar}{L}\right)\left\{-\sin \left(\varphi_{c}\right), \cos \left(\varphi_{c}\right), 0\right\}
$$

This leads to an instantaneous angular momentum caused by the quantum at position $(\boldsymbol{r})$, which is given by $\boldsymbol{S}=\boldsymbol{r} \times \boldsymbol{p}$, so that the property "spin" can be obtained simply as the average

$$
\boldsymbol{S}_{a v}=\left(\frac{1}{4 \pi^{2}}\right) \iint \boldsymbol{r} \times \boldsymbol{p} \mathrm{d} \varphi_{c} \mathrm{~d} \varphi_{t} \quad\left(\varphi_{c}, \varphi_{t} \text { from } 0 \text { to } 2 \pi\right) .
$$

The result, using (2), is $\boldsymbol{S}_{a v}=\{0,0, \hbar / 2\}$, which identifies the spin as the av- 
erage instantaneous angular momentum caused by the quantum during one period of its motion on the torus surface.

The instantaneous spin vectors during the motion form a distribution shown in Figure 3.

This distribution was shown to explain the results of spin measurements in directions forming an angle with the average spin of the particle [1], and led to the construction of our fermion model.

If the elementary charge (e) is ascribed to the quantum, the average magnetic moment caused by the rotation of the ring can be calculated. The frequency of rotation is $v=c /(L \pi)$, the well-known "Zitterbewegung" frequency. With this frequency, the current around the axis is $I=-e c /(L \pi)$. The classical expression for the magnetic moment is $\mu=I A$, with $(A)$ being the average area enclosed by the current. The average of $(A)$, is easily calculated using position vector (2) of the quantum on the torus. The result is $A_{a v}=\pi L^{2} / 2$. The magnetic moment calculated in this way becomes

$$
\mu_{a v}=-e(c / L)\left(L^{2} / 2\right)=-e c L / 2=-e \hbar /(2 m)=-\mu_{\text {classic }}
$$

This agrees with the value obtained for the Dirac electron. We point out for later use that the anomaly of the magnetic moment of the electron is given by $(\alpha / \pi=0.00232 \cdots)$, so that the magnetic moment becomes $\mu=-\mu_{\text {classic }}(1+(\alpha / \pi))$, with $(\alpha)$ being the fine structure constant.

The fermion model outlined above is completely general. The predicted properties, spin $S=\hbar / 2$, and magnetic moment, $\mu_{a v}=\mu_{\text {classic }}$, are independent of the mass, and the radius of the described particle is $R=\hbar /(m c)$. For fermions of arbitrary mass, therefore, we have the general relation

$$
R m=\hbar / c
$$

We point out that, the radius predicted by (7) depends on the relative velocity

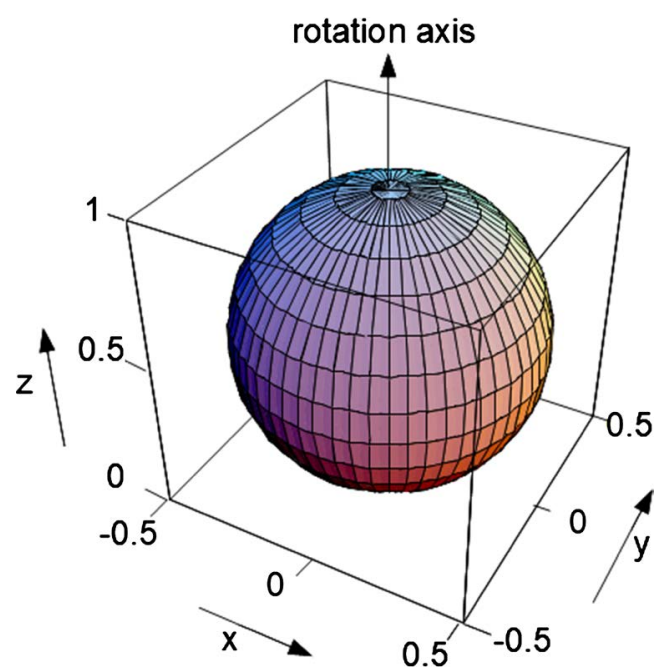

Figure 3. The instantaneous spin vector in units of $(\hbar)$ during one period of the motion of the quantum with momentum $(m c)$ around the center of the particle. The average is the observable spin vector $S_{a v}=\{0,0, \hbar / 2\}$. 
( $V)$, because the mass in the model is the relativistic mass $m=m_{0} / \sqrt{1-(v / c)^{2}}$, so that $(R)$ decreases as $R=\left(\hbar / m_{0} c\right) \sqrt{1-(v / c)^{2}}$, and reproduces the point-like character of the electron observed in scattering experiments at velocities approaching $(c)$.

\section{The Nucleons}

If we apply the general relation (7) to the mass $(M)$ of the proton, we find that the radius $R=\hbar /(M c)$ is much smaller than the recommended value. This means that, within the described fermion model, the proton is not a single fermion. Although, within the established "standard model of elementary particles"(SM), the proton is described as being composed of three quarks bound by gluons, we consider-as the most obvious possibility within our fermion model-the "merging" of two quanta of equal energy $\left(m c^{2}\right)$ into a particle of mass $(M=$ $2 \mathrm{~m}$ ). Since the "merging" leads to a topology of the paths of two quanta that does not allow to distinguish different "particles" at all, the relation to the standard model is not clear, especially, because quarks do not exist as free particles. This will be shortly discussed in paragraph 4 in the light of the results to be described below.

Below we describe how the two quanta are "fused" into a new particle of mass $(M)$ containing two quanta in periodic motions around a fixed point in space. The topology of the paths describing the synchronized motion of the two quanta, then, determines the properties of the new particle.

We consider two quanta which have equal probability on a ring in a plane perpendicular to their velocity vector. If the velocity is the velocity of light $(c)$, the distributions on the rings are static. The radius of the ring is equal to the reduced Compton wavelength $(L=\hbar /(m c)$, and therefore determines the momentum $(m c)$, and the energy $\left(m c^{2}=\hbar \omega\right)$. Each ring, with momentum ( $m c=\hbar / L)$, and speed (c) along the normal to the plane defined by the ring, represents the properties of a photon. We consider a situation, where the two quanta can "fuse". This is illustrated in Figure 4.

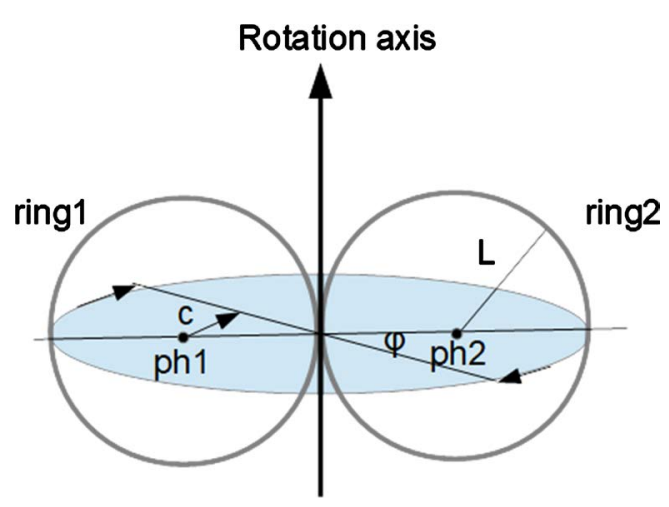

Figure 4. The situation when two photons represented by the two rings, have opposite directions, meet, and "stick together" in the way shown in the figure. The two rings then rotate as a whole around the center, which is at rest in space. The two photons, indicated as $(p h)$, keep their velocity $(c)$, and rotate on circles of radius $(L)$ with frequency $v=$ $c /(2 \pi L)$. The radius of the "entity" formed is $R=2 L$. 
The situation is described in the legend of the figure. The position vectors of the two quanta on the two rings are $\boldsymbol{r}_{1}=\{x, y, z\}$, and $\boldsymbol{r}_{2}=\{-x,-y, \pm z\}$, and are given by relation (2), except that $(L)$ has to be replaced by $(2 L)$ :

$$
\begin{gathered}
\boldsymbol{r}_{1}=2 L\left\{\cos ^{2}\left(\frac{\varphi_{t}}{2}\right) \cos \left(\varphi_{c}\right), \cos ^{2}\left(\frac{\varphi_{t}}{2}\right) \sin \left(\varphi_{c}\right), \sin \left(\varphi_{t}\right) / 2\right\} \\
\boldsymbol{r}_{2}=2 L\left\{-\cos ^{2}\left(\frac{\varphi_{t}}{2}\right) \cos \left(\varphi_{c}\right),-\cos ^{2}\left(\frac{\varphi_{t}}{2}\right) \sin \left(\varphi_{c}\right), \pm \sin \left(\varphi_{t}\right) / 2\right\}
\end{gathered}
$$

The common torus formed in the periodic motion of the two quanta represents an "entity" of energy $M c^{2}$, and of radius $2 L=4 \hbar / M c$ at rest in space. We identify this torus with the neutron. A parametric plot of the positions of the two quanta using relations (8) is shown in Figure 5. The nucleon radii predicted in this way are:

$$
\begin{gathered}
R_{\text {neutron }}=2 L=4 \hbar / M c=0.84007 \cdots \mathrm{fm} \\
R_{\text {proton }}=0.84123 \cdots \mathrm{fm}
\end{gathered}
$$

This proton value is-within the limits of error-equal to the most recent value for the charge radius of the proton, which has been obtained from an evaluation of Lamb-shift measurements on myonic hydrogen using quantum electrodynamic methods [11]. To our knowledge our result (9a) is the only purely theoretical prediction available today. Therefore, if the complete agreement between the value given in (9a), and the experimental result, is accepted to be not "accidental", it has to be concluded that the "Ansatz" implied in our fermion model, which is alternative to quantum mechanics and standard model, merits further attention.

The spin caused by the common motion of the two quanta can be obtained by defining the relative coordinate as $\boldsymbol{R}=\boldsymbol{r}_{1}-\boldsymbol{r}_{2}$, and a reduced mass $m_{1} m_{2} /\left(m_{1}+m_{2}\right)=m / 2$. For these reduced quantities, the momentum becomes, $\boldsymbol{P}=\hbar /(4 L)\left\{-\sin \left(\varphi_{c}\right), \cos \left(\varphi_{c}\right), 0\right\}$, and the instantaneous spin is $\boldsymbol{S}=\boldsymbol{P} \times \boldsymbol{R}$.

The average spin calculated for $(R)$, and $(-R)$, using expression (8) becomes

$$
S_{\text {proton }}=\{0,0, \hbar / 2\} \text { and }\{0,0,-\hbar / 2\}
$$

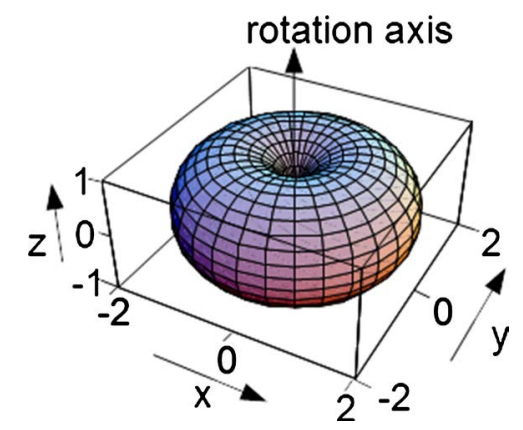

Figure 5. Representation of the nucleon by the possible positions of the two quanta during their periodic motion. The coordinates are given in units of $(L)$. The instantaneous positions of the two quanta are on opposite sides of the axis, and the radius of the nucleon is four times its reduced Compton wavelength. 
Assigning a charge $(Q)$ to the position $\left(\boldsymbol{R}=\boldsymbol{r}_{1}-\boldsymbol{r}_{2}\right)$, we obtain from the general relation $\mu=I A$, with the current $I=Q c /(4 \pi L)$, and the average surface $(A)$ calculated with $(8,8 \mathrm{a})$ two different magnetic moments, depending on the $(+)$-, or (-)-sign chosen in (8a) for the z-coordinate. We obtain, respectively,

$$
\begin{aligned}
& \mu(a)=6(Q / e) \mu_{n} \\
& \mu(b)=8(Q / e) \mu_{n}
\end{aligned}
$$

In $(11),\left(\mu_{n}\right)$ is the classical nuclear magnetic moment.

We have not investigated the role of the quantity "charge" in the context of our model. We point out, however, that with $Q / e= \pm 1 / 3$ one obtains possible magnetic moments of

$$
\mu(a)= \pm 2 \mu_{n}
$$

The ( \pm )-signs we ascribe to the possible formation of particle and anti-particle. The magnetic moment for the particle neutron is negative. If this required (-) sign is chosen, $\mu(a)=-2 \mu_{n}$ deviates by only ca. $5 \%$ from the established value of the neutron magnetic moment of $\mu_{\text {neutron }}=-1.91 \cdots \mu_{n}$. We therefore conclude that the model predicts

$$
\mu_{\text {neutron }}=-2 \mu_{n}
$$

The total charge of the neutron is zero, so that for the charges $\left(q_{1}\right)$ and $\left(q_{2}\right)$ of the quanta relation $q_{1}+q_{2}=0$ is required. With the condition $\boldsymbol{R}=\boldsymbol{r}_{1}-\boldsymbol{r}_{2}$, which implies $Q=q_{1}-q_{2}=-(1 / 3) e$, we thus obtain $q_{1} / e=-1 / 6$ and $q_{2} / e=1 / 6$, for the neutron. In addition we point out that the $(+)$-sign in relation (8) characterizes the neutron (antineutron).

The proton magnetic moment has the (+)-sign, and by loss of one electron from the neutron, the total charge increases to $+1 e$. For symmetry reasons we assume that the extra charge $(e)$ is added equally to the charges $q_{1}=-(1 / 6) e$ and $q_{2}=(1 / 6) e$, leading to $q_{1}=(1 / 3) e$, and $q_{2}=(2 / 3) e$, for the proton. The required positive $(Q)$ can only be obtained for $Q=q_{2}-q_{1}=(1 / 3) e$, which corresponds to the topology given by $\boldsymbol{R}=\boldsymbol{r}_{2}-\boldsymbol{r}_{1}$. This shows that the formation of a proton proceeds by loss of an electron from an anti-neutron. Relation (11), and $Q=$ $(1 / 3) e$ lead to $\mu(b)=(8 / 3) \mu_{n}$. Since this value deviates by only ca. $5 \%$ from the established value of the proton magnetic moment of $2.79 \ldots \mu_{n}$, we therefore conclude that the model predicts a value of the proton magnetic moment of

$$
\mu_{\text {proton }}=\mu(b)=(8 / 3) \mu_{n}=2.67 \mu_{n}
$$

We point out that the proton in this way is characterized by the (-)-sign in relation (8), so that the proton topology differs from the neutron topology. Within the model, therefore, the transition (anti-neutron $\rightarrow$ proton $+\mathrm{e}^{-}$) is accompanied by a topology change. This topology change probably characterizes the loss of an antineutrino implied in the transition.

We expect the values $(13,14)$ to be correct at the level where the magnetic moment for the fermion becomes equal to $\mu_{\text {classic }}$. For the neutron we derive a correction factor caused by the $(g-2)$ anomaly by comparing the predicted value 
(13), with the recommended value of $1.9130 \mu_{n}$, as $k=1-1.9130 / 2=0.0435$. For the proton formed from the anti-neutron by loss of an electron we include the established correction factor of (0.00232), and obtain

$$
\mu_{\text {proton }}=(8 / 3)(1+0.0435+0.00232) \mu_{n}=2.7888 \mu_{n},
$$

which agrees very closely with the recommended value. To our knowledge, a calculation from first principles of the magnetic moments of the nucleons is not yet available.

\section{Summary}

We have shown in this paper that our extended fermion model is able to predict the proton radius in "complete" agreement with the most recent experimental value of the charge radius. The radius predicted is-simply-four times the reduced Compton wavelength of the proton. This result

$$
R_{\text {proton }}=4 \hbar / M c=0.84123 \cdots \mathrm{fm}
$$

is of special interest because it confirms the result of the most accurate value obtained in 2010 from an evaluation of Lamb-shift measurements on myonic hydrogen using quantum electrodynamic methods [11]. This value is

$$
R_{\text {proton }}=(0.84184 \pm 0.0007) \mathrm{fm} \text {. }
$$

Since this value disagrees with the actual recommended value, of

$$
R_{\text {proton }}=(0.8875 \pm 0.0051) \mathrm{fm},
$$

by more than the given limits of error, there existed since (2010) for some years a so-called "proton-radius puzzle". In (2019), new electron-proton scattering experiments [12], which yielded a value of

$$
R_{\text {proton }}=(0.831 \pm 0.007) \mathrm{fm} \text {, }
$$

have explained the "puzzle" as having been due to unknown experimental artefacts of earlier experiments [12]. To our knowledge, our predicted value (16) is the only theoretical value available today.

The spin $( \pm \hbar / 2)$ of the nucleons is predicted, and values of the magnetic moments of proton and neutron are obtained. These values deviate by ca. $5 \%$ from the established recommended values. This deviation we ascribe to the (g-2)-anomaly also presents for the electron. By determining the correction factor for the neutron from our predicted value (13), one obtains from (14) for the proton a predicted value of

$$
\mu_{\text {proton }}=2.7888 \mu_{n} \text {, }
$$

which is very close to the recommended value. To our knowledge, a calculation from first principles of the magnetic moments of the nucleons is not yet available.

It is remarkable that the quark "particles", invoked in the rather involved description of the nucleons within the standard model (SM), appear in the form of charges $(1 / 3) \mathrm{e}$ and $(2 / 3) e$ in our model as a consequence of the topology of the autonomous motion of two quanta. Different "particles", cannot be distin- 
guished in our model, where the topology of the paths of two quanta defines one "entity", which, for sufficiently long observation times, can be viewed as one particle, in the present case as one of the nucleons. The validity of the standard model, and of the quantum chromodynamics treatment of the nuclei is, of course, not questioned. On the other hand, the successes of the fermion model, demonstrated for the electron in references [1] [2] [3], and for the nucleons in this paper, suggest that there exists a close relation between the two descriptions, which deserves further investigations.

The outline given of the model shows that, it follows from an "Ansatz" which is "alternative" to quantum mechanics and (SM): instead of "particles", quanta are the basis, which form photons, fermions, and nucleons, in autonomous periodic motion. With this description, the nucleons are not composed of particles, but are rather "elementary", in a similar way as fermions are. These elementary particles have internal structure and can own properties. For their radius $(R)$ we have the general relation

$$
R M=4(\hbar / c),
$$

with $(M)$ being the relativistic mass, which implies a velocity dependence of the radius.

The model is completely general because it does not involve any free parameters.

\section{Conflicts of Interest}

The author declares no conflicts of interest regarding the publication of this paper.

\section{References}

[1] Niehaus, A. (2016) Foundations of Physics, 46, 3-13. https://doi.org/10.1007/s10701-015-9953-y

[2] Niehaus, A. (2017) Journal of Modern Physics, 8, 511-521. https://doi.org/10.4236/jmp.2017.84033

[3] Niehaus, A. (2019) Journal of Modern Physics, 10, 423-431. https://doi.org/10.4236/jmp.2019.104027

[4] Schrödinger, E. (1930) Akad. Wiss. Phys.-Math. K., 24, 418.

[5] Hestenes, D. (1990) Foundations of Physics, 20, 1213-1232. https://doi.org/10.1007/BF01889466

[6] Hestenes, D. (2003) Annales de la Fondation Louis de Broglie, 28, 390-408.

[7] Weyssenhoff, J. (1947) Acta Physica Polonica, 9, 47.

[8] Barut, A.O. and Sanghi, N. (1984) Physical Review Letters, 52, 2009-2012. https://doi.org/10.1103/PhysRevLett.52.2009

[9] Pavsic, M., Recami, E., Waldyr, A., Rodriges Jr., G., Maccarrone, D., Racciti, F. and Salesi, G. (1993) Physics Letters B, 318, 481-488. https://doi.org/10.1016/0370-2693(93)91543-V

[10] Wall, E.L. (2016) Hadronic Journal, 39, 71.

[11] Pohl, R., Antognini, A., Nez, F., et al. (2010) Nature, 466, 213-216. https://doi.org/10.1038/nature09250

[12] Xiong, W., Gasparian, A., Zhao, Z.W., et al. (2019)Nature, 575, 147-150. 